\title{
SUBCRITICAL HYDROTHERMAL CONVERSION (SHC) PROCESS SUPPORTED BY MICROWAVES
}

Ryszard Parosa

PROMIS-TECH, Poland

\section{Introduction}

The process described in this article uses the change of the property of water in the socalled critical state in very high pressure - about 22,131,360 Pa (over $200 \mathrm{~atm}$ ). In the critical state, water becomes a very effective solvent, causing the biomass to break down into simple hydrocarbons, including mainly bio-diesels (liquid hydrocarbons), gases (methane, ethane, etc.), and the so-called bio-char. The process is illustrated in fig. 1.

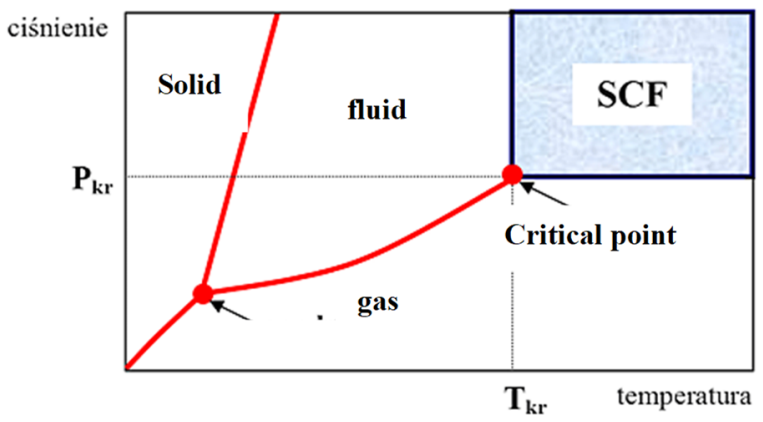

Fig. 1. Graph

Until now, the process in question has been used in a system with conventional heating of the mass being utilised through heating the walls of the high-pressure chamber. It requires the heating of thick metal walls and the material inside needed a long heating time as a result of thermal conductivity.

The essence of the process in question is to use microwave energy for controlled maintenance (stabilisation) of temperature in the process chamber in which the biomass is fed under very high pressure. In critical conditions, water ceases to be a polar liquid and dielectric parameters of water change rapidly (dielectric permeability and the so-called loss angle tangent). Thanks to that property, the introduction of microwaves (in the frequency band from $900 \mathrm{MHz}$ to $2.5 \mathrm{GHz}$ ) into the process chamber makes it possible to precisely maintain critical conditions through the control of the reflected wave in the microwave line. The essence of the process is presented in the flow chart (below). 


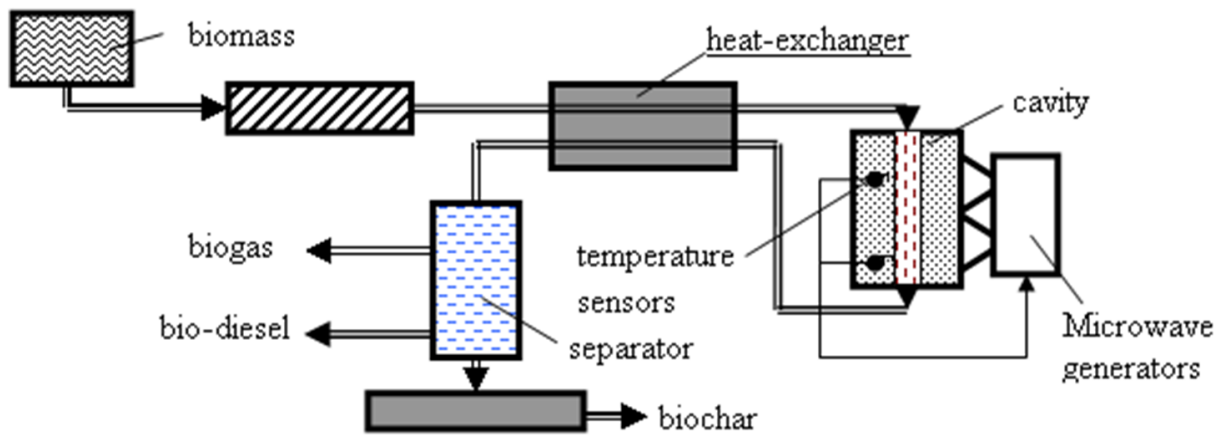

Fig. 2.

The biomass (sludge) is pressed into the heat exchanger, where it is initially heated and then fed to the high-pressure process chamber, where the biomass is heated up to required high temperature of about $260-300^{\circ} \mathrm{C}$. After hydrothermal conversion of the biomass in the process chamber, the products of conversion are pressed through the heat exchanger, in which they are cooled down, and the recovered thermal energy is used for the heating of another portion of biomass to be utilised. Cooled down products of conversion of the biomass are then separated into gaseous fractions (biogas), liquid fractions (bio-diesel), and solid fractions (biochar).

The use of microwave energy is aimed at enhancing (accelerating) the process of heating of biomass, ensuring uniform heating throughout the volume (and not only along the walls, as is the case in the conventional method).

The implementation process is divided into three stages:

- tests in a laboratory scale for the determination of optimal process conditions;

- studies and tests in a semi-technical installation;

- construction of a prototype industrial installation.

Tests in a laboratory scale with the use of a metal chamber fitted with a connection allowing microwaves to be introduced inside the chamber.

The laboratory installation was supplied from the microwave generator with frequency of $2.45 \mathrm{GHz}$ and continuous regulated power in the range from $300 \mathrm{~W}$ to $3 \mathrm{~kW}$. In the microwave supply line, a reflectance mater was installed for the measurement of reflected power, together with a ferrite circulator (cf. figure 3). 


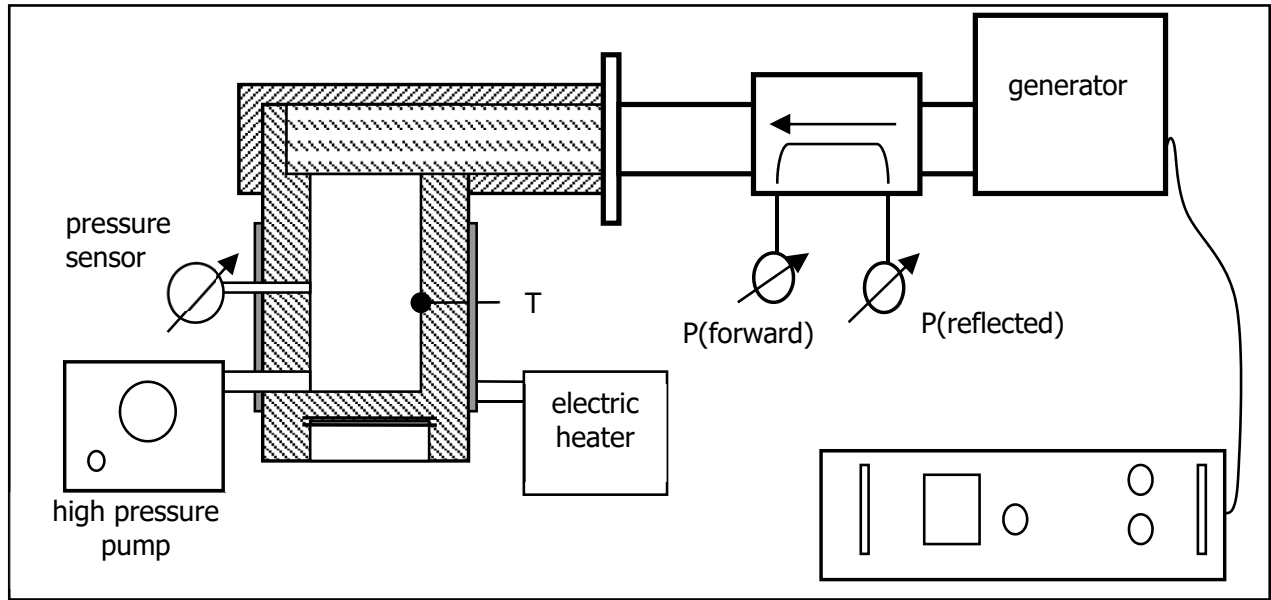

Fig. 3.

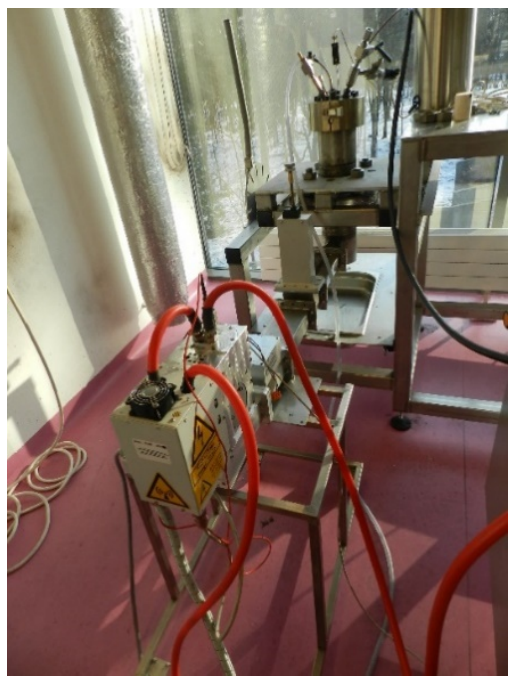

Fig. 4.

In the photograph, a laboratory installation is shown. The process was conducted with stabilised sludge with initial moisture of 50\%-70\%.

A series of tests allowed the determination of optimal conditions of the process, i.e. pressure, temperature in the process chamber, and microwave heating time — in order to obtain as large volume of liquid as possible with composition including flammable hydrocarbon substances. Examples of results are presented in table 1. 


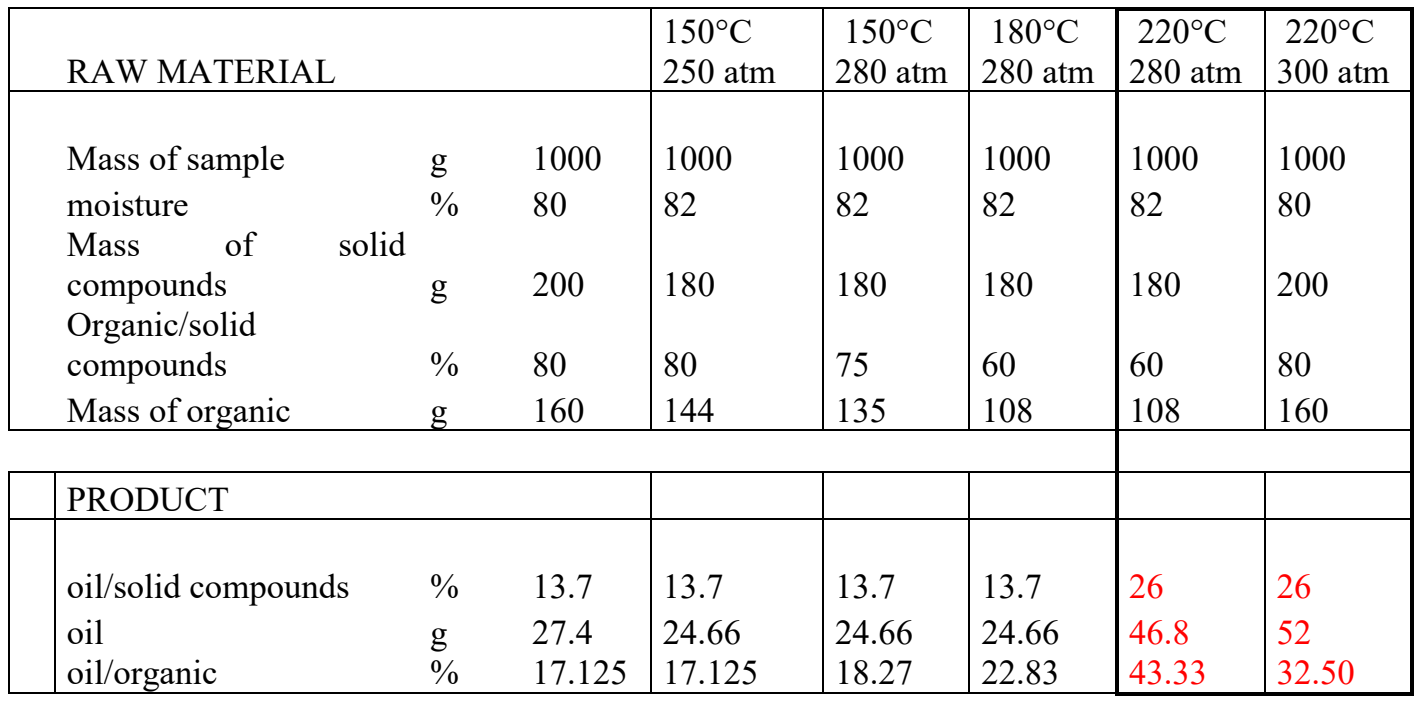

Based on the results of tests conducted on the laboratory installation, a semi-technical installation was developed. The installation was installed inside the standard container cf. photographs.

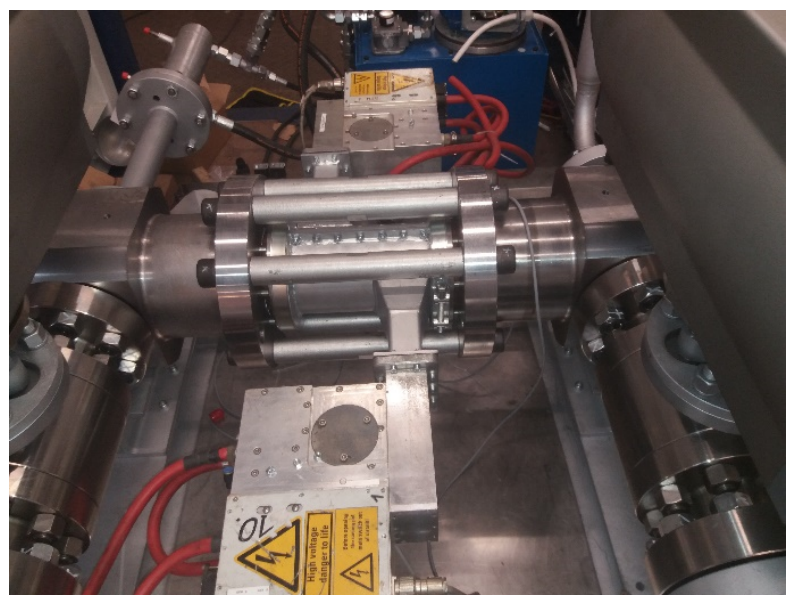

Fig. 5. 


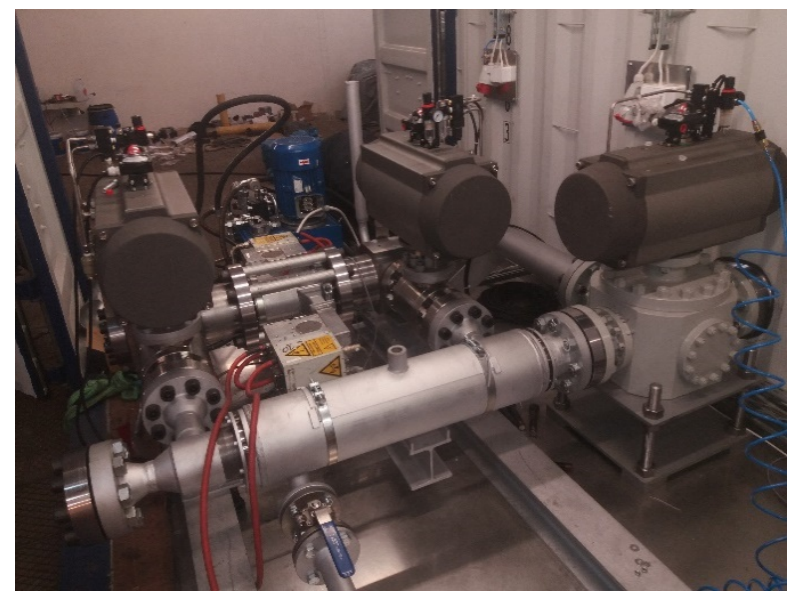

Fig. 6.

The high-pressure chamber was made of zirconium ceramics (pipe $\Phi 140 \mathrm{~mm} / \mathrm{ext}$.) with a metal cover, on which two waveguide radiators were installed, connected with generators of $3 \mathrm{~kW}(\mathrm{CW})$ power each $(\mathrm{f}=2.45 \mathrm{GHz})$. The structure with a high-pressure pump and a system of valves allowed the obtainment of required pressure in the process chamber. Before being fed to the process chamber, the processed sludge was pre-heated to a temperature of over $160^{\circ} \mathrm{C}$. Further heating was performed with the use of microwaves. In the course of the heating process, thermal insulation aimed at reducing energy losses was placed on the metal cover, and in further experiments an additional electric heater was installed in order to accelerate the process of heating of sludge. As a result, sludge was heated in the area next to the walls of the process chamber due to the absorption of microwaves and additionally due to thermal conductivity.

In the course of the process, on one microwave line between the generator and the radiator, a microwave reflectance meter was installed, and the size of the wave reflected during the heating process was recorded. A small increase of the reflected wave after the heating of sludge to the temperature of $200^{\circ} \mathrm{C}$ was found. With transmission of microwaves of power of about $2980 \mathrm{~W}$ into the process chamber, the power of the reflected wave changed from about $130 \mathrm{~W}$ to slightly over $160-180 \mathrm{~W}$ after heating the sample to a temperature of $200^{\circ} \mathrm{C}$. It was connected with changes in dielectric parameters of the material in the process chamber after achieving conditions close to the critical state.

Tests in that installation allowed the obtainment of up to 6 litres of bio-oil in one cycle of about 30-40 minutes. The technological problem is the selection of material for the highpressure pipe that would be transparent to microwaves. The use of zirconium pipes proved suboptimal - such pipes absorbed a part of the microwave energy, heating up unevenly, mainly directly next to microwave radiators. Those experiences have led to a search for another method of transmission of microwaves to the biomass being heated up in relation to previously planned method presented in the figure below. 


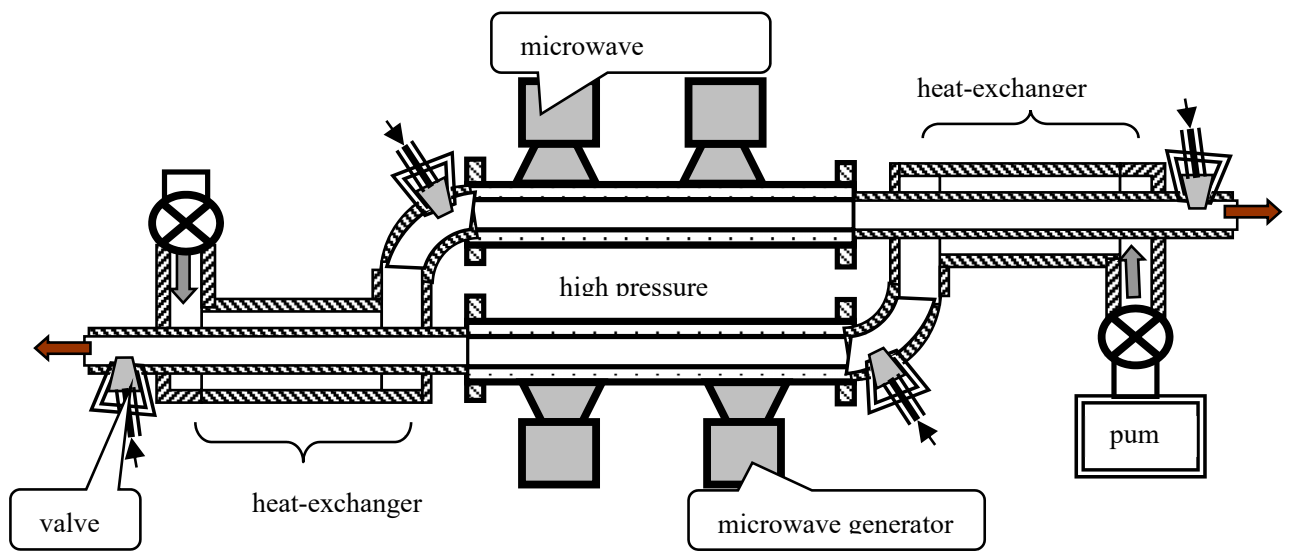

Fig. 7.

The structure of the industrial installation being currently prepared assumes the use of rod microwave radiators placed inside the metal high-pressure chamber. This will make it possible to use alund ceramics for the cover of radiators and to eliminate inconveniences connected with the use of zirconium pipes.

\section{Summary:}

It has been confirmed that the described process of application of microwave energy for utilisation of biomass with the method of hydrothermal conversion is practicable in laboratory conditions and in an industrial scale. It is confirmed by results of tests and an initial analysis of viability of the process. However, it is necessary to remove technical inconveniences connected with the problem of lossless transmission of microwaves through special ceramic materials and to ensure the most uniform distribution of the electromagnetic field inside the process chamber. Such works are being continued and a new reactor in a technical scale is being designed. The reactor is intended to make it possible to carry out a process competitive to known conventional methods of utilisation of sludge. 